\title{
Guatiní: un proyecto para fomentar el conocimiento de la avifauna cubana en las nuevas generaciones
}

Guatiní: A Project to promote the knowledge of the endemic birds of Cuba in the new generations

\author{
Juan Carlos Sepúlveda-Peña ${ }^{1}$ \\ Bislandry Vejo-Paula ${ }^{2}$ \\ Carlos Villanueva-Hedesa ${ }^{3}$ \\ Juan Ramón González-Guerra ${ }^{4}$ \\ Alieny González-Alfonso ${ }^{5}$ \\ lanela García-Lau ${ }^{6}$ \\ Gladis Hernández-Horta ${ }^{7}$ \\ Manuel Borrego ${ }^{8}$ \\ Gilberto Socorro ${ }^{9}$ \\ Alberto Amat-Álvarez ${ }^{10}$ \\ Maritza González-Moreno ${ }^{11}$
}

Fecha de recepción: 9 de septiembre de 2018

Fecha de aprobación: 2 de diciembre de 2018

\section{Resumen}

En Cuba existen 29 especies de aves amenazadas o casi amenazadas, tres en peligro crítico, ocho en peligro, siete vulnerables, y once cercanas a la amenaza. Además de la pérdida y degradación de los hábitats, las aves de Cuba se ven amenazadas por la cacería, la colecta de huevos y el comercio ilegal. Revertir esta situación va más allá de las leyes y acciones de las autoridades competentes, es necesaria una educación medioambiental desde las edades tempranas. Se necesita que los individuos y las colectividades se identifiquen con su entorno y adquieran los conocimientos, los valores, los comportamientos y las habilidades para participar responsable y eficazmente en la prevención y la solución de los problemas ambientales. En la actualidad, niños y jóvenes hacen un gran uso de los teléfonos móviles, tabletas y computadoras principalmente para jugar; en su mayoría, estos juegos solo entretienen y no educan, y en algunos casos se podría decir que enajenan. ¿Por qué no aprovechar estas tecnologías para incentivar desde edades tempranas el conocimiento

\footnotetext{
${ }^{1}$ Ph. D. Universidad Tecnológica de La Habana “José Antonio Echeverría” (La Habana, Cuba). icarlos@ceis.cujae.edu.cu.

2 Universidad Tecnológica de La Habana “José Antonio Echeverría” (La Habana, Cuba). bvejo@ceis.cujae.edu.cu.

${ }^{3}$ Universidad Tecnológica de La Habana "José Antonio Echeverría” (La Habana, Cuba).

${ }^{4}$ Universidad Tecnológica de La Habana "José Antonio Echeverría” (La Habana, Cuba).

${ }^{5}$ M. Sc. Universidad de La Habana (La Habana, Cuba). aglez@fbio.uh.cu.

${ }^{6}$ M. Sc. Universidad de La Habana (La Habana, Cuba). ianela@fbio.uh.cu.

7 Escuela "José de la Luz Caballero" (La Habana, Cuba).

${ }^{8}$ Refugio de Fauna Sureste del Inglés (Mayabeque, Cuba).

${ }^{9}$ Refugio de Fauna Sureste del Inglés (Mayabeque, Cuba).

10 Universidad Tecnológica de La Habana "José Antonio Echeverría" (La Habana, Cuba).

${ }^{11}$ Universidad Tecnológica de La Habana “José Antonio Echeverría” (La Habana, Cuba). marmel@tesla.cujae.edu.cu. ORCID: 0000-0001-5646-2114.
} 
y el amor por la flora y la fauna? El proyecto Guatiní tiene como objetivo desarrollar juegos y aplicaciones multimedia que permitan divulgar la información científica acumulada sobre la avifauna cubana y educar así principalmente a las nuevas generaciones en el cuidado del medio ambiente.

Palabras clave: aves endémicas; ecología; educación; medio ambiente; móvil.

\section{Abstract}

In Cuba there are 29 species of threatened or almost threatened birds, three of them in critically endangered, eight in danger, seven vulnerable, and eleven close to the threat. In addition to the loss and degradation of habitats, Cuban birds are threatened by hunting, egg collection and illegal trade. Reversing this situation goes beyond the laws and actions of the competent authorities, an environmental education is necessary from an early age. It is necessary that individuals and communities understand the complex nature of the natural environment and acquire the knowledge, values, behaviors and skills to participate responsibly and effectively in the prevention and solution of environmental problems. Nowadays children and young people make a great use of mobile phones, tablets and computers mainly to play; these games mostly just entertain and do not educate, and in some cases we could say that they alienate. Why not take advantage of these technologies with the aim of encouraging early knowledge and love for flora and fauna? The goal of the Guatini project is the development of games and multimedia applications that allow the dissemination of the accumulated scientific information on the Cuban avifauna and thus educate mainly the new generations in the care of the environment.

Keywords: ecology; education; endemic bird; environmental; mobile.

Para citar este artículo:

Sepúlveda-Peña, J. C., Vejo-Paula, B., Villanueva-Hedesa, C. A., González-Guerra, J. R., González-Alfonso, A., García-Lau, I., Hernández-Horta, G., Borrego, M., Socorro, G., Amat, A., \& González-Moreno, M. (2019). Guatiní: un proyecto para fomentar el conocimiento de la avifauna cubana en las nuevas generaciones. Ciencia y Agricultura 16(1), 17-30. DOI: https://doi.org/10.19053/01228420.v16.n1.2019.8803.

Esta obra está bajo licencia internacional Creative Commons Reconocimiento 4.0 
Juan Carlos Sepúlveda-Peña, Bislandry Vejo-Paula, Carlos A. Villanueva-Hedesa, Juan R. GonzálezGuerra, Alieny González-Alfonso, lanela García-Lau, Gladis Hernández-Horta, Manuel Borrego, Gilberto Socorro, Alberto Amat, Maritza González-Moreno

\section{Introducción}

La localización geográfica de Cuba no solo la convierte en un punto estratégico para los países americanos, su posición en el Caribe la erige en un importante corredor migratorio y sitio de invernada para un gran número de aves migratorias neotropicales. Cuba se encuentra en las rutas migratorias de la cuenca del Mississippi y de la costa atlántica de Norteamérica, y miles de rapaces, patos, playeras y aves terrestres atraviesan todos los años el país. El Caribe es reconocido como el quinto Hotspot del planeta por su importancia para la conservación de la biodiversidad. Esta área alberga más de 500 especies de aves, incluidas 172 especies endémicas (Birdlife.org, 2017).

El archipiélago cubano es extremadamente importante para las aves acuáticas, y su red de humedales naturales y artificiales proveen un hábitat crítico para las mayores concentraciones de especies registradas en el Caribe, siendo Cuba la isla del Caribe de mayor diversidad biológica y con mayor diversidad de aves en el Caribe insular (Raffaele, Garrido, Keith \& Raffaele, 2003), donde se presentan altos niveles de endemismo; por ejemplo, más del $32 \%$ de los vertebrados son endémicos (Aguilar-Mujica, Díaz-Fernández, Clay, Davidson \& Yépez-Zabala, 2009). En Cuba actualmente existen 29 especies de aves amenazadas o cercanas a la amenaza, tres de ellas en peligro crítico (gavilán caguarero, Chondrohierax wilsonii; carpintero real, Campephilus principalis y bijirita de Bachman, Vermivora bachmanii), ocho en peligro, siete vulnerables, y 11 cercanas a la amenaza (Aguilar-Mujica et al., 2009).

Además de la pérdida y degradación de los hábitats, las aves de Cuba se ven amenazadas por la cacería, la colecta de huevos y el comercio ilegal (AguilarMujica, 2009; Estrada-Betancourt, 2007). Revertir esta situación va más allá de las leyes y acciones de las autoridades competentes, es necesario una educación medioambiental (Estrada-Betancourt, 2007) desde las edades tempranas. La educación ambiental debe ir dirigida a toda la humanidad, debido a que es un problema que incumbe a todos (Martínez-Castillo, 2010), incluyendo los niños y jóvenes. Su importancia consiste en lograr que los individuos y las colectividades incrementen su conocimiento sobre el ambiente 
natural y del creado por el ser humano, y adquieran los conocimientos, los valores, los comportamientos y las habilidades para participar responsable y eficazmente en la prevención y la solución de los problemas ambientales (Martínez-Castillo, 2010).

En una investigación realizada por los autores en diez librerías y tres bibliotecas de la capital se pudo constatar la poca disponibilidad de libros relacionados con las aves endémicas y su protección. De los 13 sitios visitados, solamente se pudo encontrar bibliografía referida a las aves endémicas de Cuba en la Biblioteca Nacional para su consulta in situ.

En el caso de las aplicaciones multimedia y los juegos didácticos, se encontraron seis aplicaciones, algo antiguas, en la Biblioteca Nacional de Cuba, de las cuales ninguna era compatible con los sistemas operativos modernos. Durante la investigación acerca de las aplicaciones multimedia existentes sobre la avifauna cubana, se encontraron solo dos aplicaciones desarrolladas por la empresa CITMATEL, disponibles para sistema operativo Windows.

Por otro lado, "los dispositivos móviles se han convertido en protagonistas de la vida diaria de las personas. En el caso del teléfono móvil, su crecimiento a finales de la primera década del siglo XXI, lo había convertido en el medio de comunicación más extendido del mundo, superando a la prensa escrita, la televisión e internet. La presencia social de estos dispositivos móviles es diversa, incluyendo contextos de educación y formación, y la explotación de sus funcionalidades y prestaciones técnicas con fines didácticos ha dado lugar al surgimiento de una nueva modalidad educativa: el mobile learning o aprendizaje móvil" (Brazuelo \& Cacheiro, 2015).

Los videojuegos han logrado ocupar un importante lugar en la vida de los niños y jóvenes, incluso algunos autores afirman que "la familia juega delante de la pantalla a través de consolas que han acercado el juego al hogar. Este fenómeno hace que sea cada vez más difícil dejar de lado el análisis de los usos educativos de los juegos" (Salvat, 2009). Es por estas razones que los autores del presente artículo decidieron aprovechar las prestaciones y 
Juan Carlos Sepúlveda-Peña, Bislandry Vejo-Paula, Carlos A. Villanueva-Hedesa, Juan R. González-

Guerra, Alieny González-Alfonso, lanela García-Lau, Gladis Hernández-Horta, Manuel Borrego, Gilberto

Socorro, Alberto Amat, Maritza González-Moreno

fortalezas de los ordenadores y dispositivos móviles para dar soporte a aplicaciones multimedia y juegos que ayuden en la educación medioambiental, específicamente en lo relacionado con el conocimiento de la avifauna cubana.

Según Lukin (2014), para el desarrollo de actividades de educación ambiental se ha definido una serie de tipos de juegos, entre los que se cuentan juegos de presentación, juegos de simulación, juegos ambientales y juegos de conocimientos del entorno y de sensibilización. El proyecto Guatiní se centró en el desarrollo de este último tipo de juego, además del desarrollo de una aplicación multimedia que fuera posible ejecutarla en diferentes plataformas de hardware como PC, tabletas y smartphone, e igualmente en diferentes plataformas de software, tales como Windows, Linux y Android.

La mayor amenaza que enfrenta la biodiversidad en la actualidad es la pérdida de hábitat. Las aves no escapan a esta amenaza y como consecuencia algunas se encuentran dentro de la lista roja de la UICN. Para algunas regiones como el Caribe, esta situación es alarmante por el alto grado de endemismo que existe. En Cuba se registran 27 especies de aves endémicas (Aguilar-Mujica et al., 2009; Estrada-Betancourt, 2007; Navarro-Pacheco, 2015) que representan el 7 \% de la avifauna cubana, de las cuales 19 están bajo algún nivel de amenaza; una de ellas reportada como extinta (guacamayo cubano).

En la actualidad, el conocimiento sobre la avifauna cubana se concentra en libros impresos de reducida tirada. A ellos solo se puede acceder a través de las bibliotecas públicas del país, además de las universidades. Con la aparición de las tecnologías de la comunicación y la informatización (TIC) es posible que este conocimiento llegue a una mayor cantidad de personas y es en este aspecto en que asiste la Facultad de Ingeniería Informática de la Universidad Tecnológica de La Habana (CUJAE) a la promoción cultural de las aves endémicas mediante recursos que sistematicen la información recopilada por especialistas e interesados como la comunidad a la que responde el presente proyecto. 
Como resultado de este proyecto, mediante recursos tecnológicos se socializan conocimientos sobre el tema aludido con el objetivo de satisfacer las necesidades sentidas de la comunidad, cuya población estratificada (niños de edad primaria de la Escuela José de la Luz y Caballero, Consejo Popular "Los Palos", municipio de Nueva Paz, provincia Mayabeque, miembros del proyecto de educación ambiental "Guanamón"; especialistas de flora y fauna del refugio de fauna Sureste del Inglés, profesores del Grupo de Ecología de Aves de la Facultad de Biología de la Universidad de La Habana y estudiantes de la Facultad de Ingeniería Informática de la CUJAE), exige sistematización y promoción cultural sobre el mundo de las aves y la importancia de conservar sus hábitats. El trabajo educativo con las nuevas generaciones puede ser una de las principales acciones para crear conciencia sobre el problema y así revertir las circunstancias de amenaza a la biodiversidad (Estrada-Betancourt, 2007).

En Cuba, al igual que en muchas partes del mundo, el desarrollo humano ha ido en detrimento de los hábitats de especies animales (Aguilar-Mujica et al., 2009) y vegetales, muchas de ellas con un endemismo local (EstradaBetancourt, 2007). En la población cubana, en general, no hay conciencia de este problema, a pesar de la divulgación a través de los medios de difusión masiva. Sin embargo, esto no es suficiente. Para llevar este conocimiento y problemática a las nuevas generaciones es imprescindible usar también las TIC, las cuales son herramienta de gran ayuda dada su utilización por parte de los jóvenes. En la actualidad, niños y jóvenes hacen un gran uso de los teléfonos móviles, tabletas y computadoras, principalmente para jugar; juegos que en su mayoría solo entretienen y no educan, y en algunos casos se podría decir que enajenan. Por tanto, también es un problema el uso indiscriminado que hacen los jóvenes de estas tecnologías sin un fin productivo o educativo, y en algunos casos hasta nocivos para la salud (Vanderwater, Wartella, Huang, Lee \& Shim, 2007; Gailey, 1993). En la literatura ya se reportan adicciones a este tipo de juegos (Vanderwater et al., 2007; Gailey, 1993) que no les aporta nada como individuos. Por qué no aprovechar entonces estas tecnologías con el objetivo de incentivar desde edades tempranas el amor por la flora y la fauna, y contribuir de esta forma a la formación integral de los estudiantes 
Juan Carlos Sepúlveda-Peña, Bislandry Vejo-Paula, Carlos A. Villanueva-Hedesa, Juan R. González-

Guerra, Alieny González-Alfonso, lanela García-Lau, Gladis Hernández-Horta, Manuel Borrego, Gilberto Socorro, Alberto Amat, Maritza González-Moreno

desde el nivel primario de enseñanza hasta el nivel universitario e incluso postgraduado.

\section{Materiales y métodos}

Los autores estudiaron las aplicaciones para dispositivos móviles existentes en el ámbito nacional e internacional y solamente encontraron aplicaciones con información de avifaunas foráneas, pero no sobre las aves endémicas de Cuba.

Sin embargo, la comunidad antes mencionada, inquieta por una misma necesidad: crear recursos informáticos para la promoción cultural de la avifauna cubana, solicita a la CUJAE su acompañamiento para lograr este propósito. Esta necesidad sentida queda diagnosticada mediante los siguientes métodos: observación participativa, con participación activa; entrevista informal; análisis documental y estudio de literatura científica.

A partir del estudio realizado se determinó como problema la insuficiente divulgación de la información científica acumulada sobre la avifauna cubana, por dificultades para masificar dicha información por las vías clásicas (recursos impresos) y como campo de actuación la promoción cultural medioambiental en los niveles de enseñanza primario y universitario.

Divulgar la información científica acumulada sobre la avifauna cubana mediante la creación de contenidos digitales para educar a la comunidad sobre la importancia del cuidado y conservación de las aves y sus hábitats, así como en el uso de las TIC en la masificación del conocimiento sobre medio ambiente.

Los resultados han sido divididos por etapas:

- Primera etapa: desarrollar contenido digital (multimedia, CD, DVD, etc.) para divulgar el conocimiento sobre las aves endémicas de Cuba, sus hábitats, conductas, hábitos alimentarios etc.

- Segunda etapa: migrar los contenidos digitales para un ambiente de Android, de forma que pueda ser vista desde un teléfono celular o tableta con ese sistema operativo, lo cual incluye a los futuros dispositivos decodificadores de la televisión digital terrestre ("cajitas"), 
que serán distribuidos en nuestro país. Esta migración contribuirá a la masificación de los conocimientos incluidos en la aplicación multimedia.

- Tercera etapa: desarrollar un conjunto de juegos instructivos mediante ordenador (JIMO), relacionados con las aves endémicas, de forma tal que llame la atención de los niños y jóvenes y que al jugar aprendan al mismo tiempo sobre las aves endémicas de Cuba.

- Cuarta etapa: extender los contenidos digitales, incluyendo los juegos, hasta las aves endémicas del Caribe, ya que estas, en su mayoría, también están presentes en Cuba y representan un subconjunto del total de aves de este país.

- Quinta etapa: extender los contenidos digitales, incluyendo los juegos, a todas las aves de Cuba.

Los resultados esperados tienen como objetivo promover la cultura sobre la avifauna cubana y su protección. Estos resultados podrán ser utilizados por cualquier institución docente con fines educativos, así como por cualquier otra institución sin ánimo de lucro, cuyo objetivo sea el cuidado ambiental, la ecología o la impartición de conocimientos sobre la fauna cubana.

Con el propósito de medir los conocimientos iniciales y finales de los estudiantes involucrados en el proyecto y demostrar la posible validez del uso de la aplicación multimedia y del juego didáctico en la promoción cultural sobre el tema, se hizo un diagnóstico inicial y otro final referentes al conocimiento de las aves endémicas de Cuba a un grupo de estudiantes de sexto y séptimo grado pertenecientes al proyecto Guanamón. El diagnostico consistió en una encuesta, cuyo contenido se muestra en la Figura 1.

La Tabla 1 muestra los resultados del diagnóstico inicial aplicado a 28 estudiantes de sexto y séptimo grado pertenecientes al proyecto de educación medioambiental Guanamón. En dicha tabla se indica la cantidad que respondió bien, mal, los que dejaron la pregunta en blanco y los que la respuesta estuvo bien solo a medias. Igualmente se muestran los porcentajes de las respuestas correctas (OK), mal, en blanco y correctas a medias para cada ave. 
Juan Carlos Sepúlveda-Peña, Bislandry Vejo-Paula, Carlos A. Villanueva-Hedesa, Juan R. GonzálezGuerra, Alieny González-Alfonso, lanela García-Lau, Gladis Hernández-Horta, Manuel Borrego, Gilberto Socorro, Alberto Amat, Maritza González-Moreno
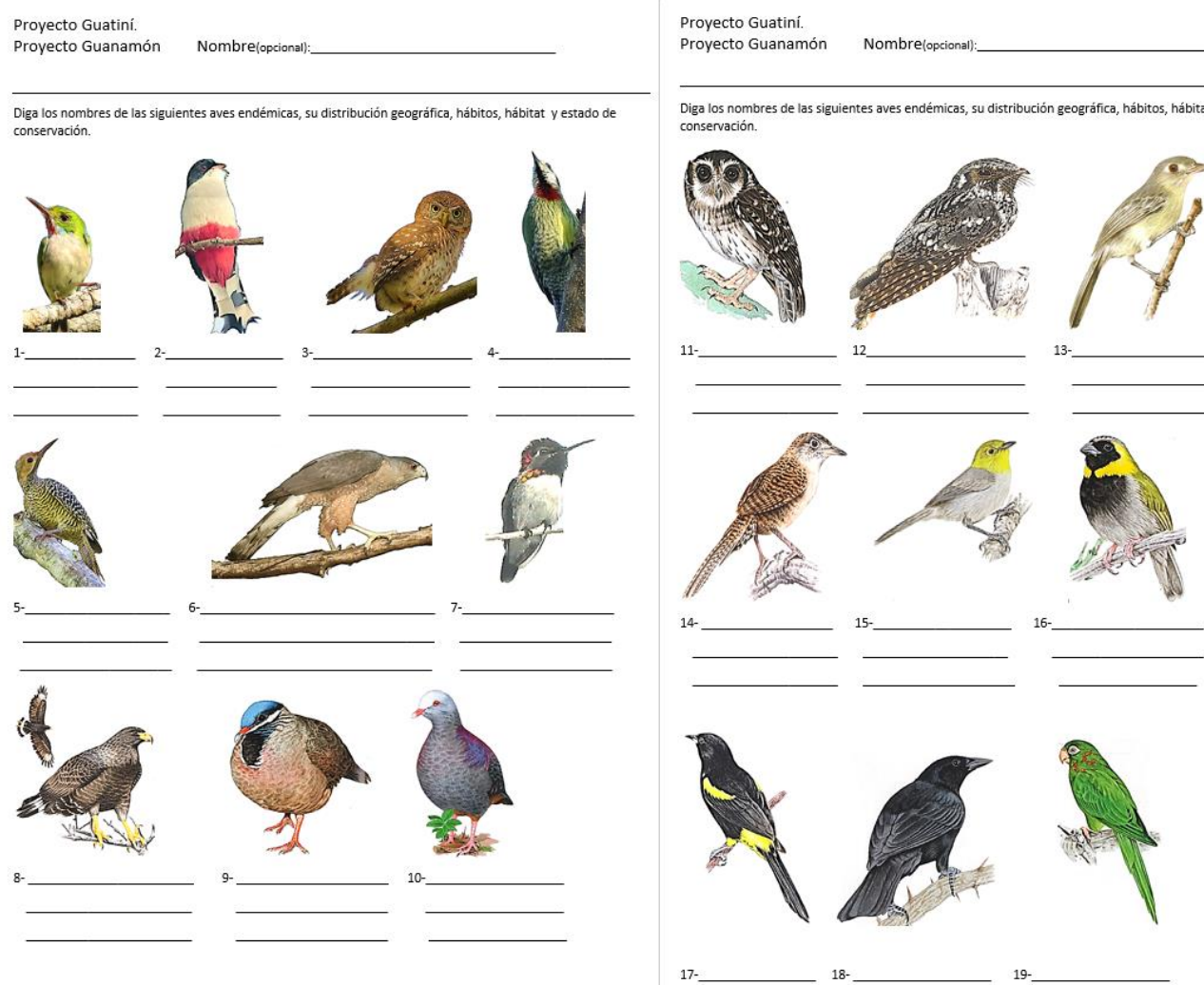

Fig. 1. Examen diagnóstico realizado.

Tabla 1. Resultado del diagnóstico inicial.

\begin{tabular}{|l|c|c|c|c|c|c|c|c|}
\hline Ave & Bien & Mal & A medias $^{*}$ & Blanco & $\%$ OK & \% OK a medias & \% Mal & \% Mal o en blanco \\
\hline Cartacuba & 12 & 4 & & 12 & 42.86 & 42.86 & 14.29 & 57.14 \\
\hline Tocororo & 28 & & & & 100.00 & 100.00 & 0.00 & 0.00 \\
\hline Sijú platanero & & 25 & & 3 & 0.00 & 0.00 & 89.29 & 100.00 \\
\hline Carpintero verde & & & 16 & 12 & 0.00 & 57.14 & 0.00 & 42.86 \\
\hline Carpintero churroso & & & 1 & 27 & 0.00 & 3.57 & 0.00 & 96.43 \\
\hline Gavilán colilargo & & 23 & 2 & 3 & 0.00 & 7.14 & 82.14 & 92.86 \\
\hline Gavilán batista & & 14 & 12 & 2 & 0.00 & 42.86 & 50.00 & 57.14 \\
\hline Zunzuncito & 1 & & 25 & 2 & 3.57 & 92.86 & 0.00 & 7.14 \\
\hline Paloma perdiz & & 8 & 2 & 18 & 0.00 & 7.14 & 28.57 & 92.86 \\
\hline Camao & & 22 & & 6 & 0.00 & 0.00 & 78.57 & 100.00 \\
\hline Sijú Cotunto & & 26 & & 2 & 0.00 & 0.00 & 92.86 & 100.00 \\
\hline Guabairo & & 5 & & 23 & 0.00 & 0.00 & 17.86 & 100.00 \\
\hline Juan Chivi Ojón & & 14 & & 14 & 0.00 & 0.00 & 50.00 & 100.00 \\
\hline Ferminia & & 6 & & 22 & 0.00 & 0.00 & 21.43 & 100.00 \\
\hline Chillina & & 6 & & 22 & 0.00 & 0.00 & 21.43 & 100.00 \\
\hline Tomeguín del pinar & 7 & 1 & 11 & 9 & 25.00 & 64.29 & 3.57 & 35.71 \\
\hline Solibio & & 7 & & 21 & 0.00 & 0.00 & 25.00 & 100.00 \\
\hline Totí & 26 & 2 & & & 92.86 & 92.86 & 7.14 & 7.14 \\
\hline Catey & & 26 & & 2 & 0.00 & 0.00 & 92.86 & 100.00 \\
\hline
\end{tabular}

${ }^{*}$ Con el término "A medias" se refiere a que solo mencionaron parte del nombre, por ejemplo, al gavilán colilargo solo lo nombraron como gavilán o al carpintero verde solo lo nombraron por carpintero.

Dos meses después del primer diagnóstico y tras haber suministrado a los estudiantes la aplicación multimedia y los juegos didácticos con la información sobre las aves endémicas, se realizó un segundo diagnóstico cuyos resultados se muestran en la Tabla 2. En este caso solamente participaron 23 estudiantes. El significado de las columnas es similar al descrito para la Tabla 1. 
Tabla 2. Resultados del segundo diagnóstico.

\begin{tabular}{|l|c|c|c|c|c|c|c|c|}
\hline Ave & Bien & Mal & A medias & Blanco & \% OK & \% OK a medias & \% Mal & \% Mal o en blanco \\
\hline Cartacuba & 22 & 1 & & & 95.65 & 95.65 & 4.35 & 4.35 \\
\hline Tocororo & 23 & & & & 100.00 & 100.00 & 0.00 & 0.00 \\
\hline Sijú platanero & 19 & & & 4 & 82.61 & 82.61 & 0.00 & 17.39 \\
\hline Carpintero verde & 18 & 1 & 4 & & 78.26 & 95.65 & 4.35 & 4.35 \\
\hline Carpintero churroso & 12 & 3 & 5 & 3 & 52.17 & 73.91 & 13.04 & 26.09 \\
\hline Gavilán colilargo & 10 & 3 & 9 & 1 & 43.48 & 82.61 & 13.04 & 17.39 \\
\hline Gavilán batista & 8 & 2 & 11 & 2 & 34.78 & 82.61 & 8.70 & 17.39 \\
\hline Zunzuncito & 21 & & 2 & & 91.30 & 100.00 & 0.00 & 0.00 \\
\hline Paloma perdiz & 5 & 4 & 12 & 2 & 21.74 & 73.91 & 17.39 & 26.09 \\
\hline Camao & 8 & 10 & & 5 & 34.78 & 34.78 & 43.48 & 65.22 \\
\hline Sijú cotunto & 12 & 5 & 5 & 1 & 52.17 & 73.91 & 21.74 & 26.09 \\
\hline Guabairo & 11 & 8 & & 4 & 47.83 & 47.83 & 34.78 & 52.17 \\
\hline Juan chivi ojón & 5 & 5 & 10 & 3 & 21.74 & 65.22 & 21.74 & 34.78 \\
\hline Ferminia & 12 & 7 & & 4 & 52.17 & 52.17 & 30.43 & 47.83 \\
\hline Chillina & 11 & 6 & & 6 & 47.83 & 47.83 & 26.09 & 52.17 \\
\hline Tomeguín del pinar & 21 & & 2 & & 91.30 & 100.00 & 0.00 & 0.00 \\
\hline Solibio & 8 & 7 & & 8 & 34.78 & 34.78 & 30.43 & 65.22 \\
\hline Totí & 23 & 0 & & & 100.00 & 100.00 & 0.00 & 0.00 \\
\hline Catey & 14 & 6 & & 3 & 60.87 & 60.87 & 26.09 & 39.13 \\
\hline
\end{tabular}

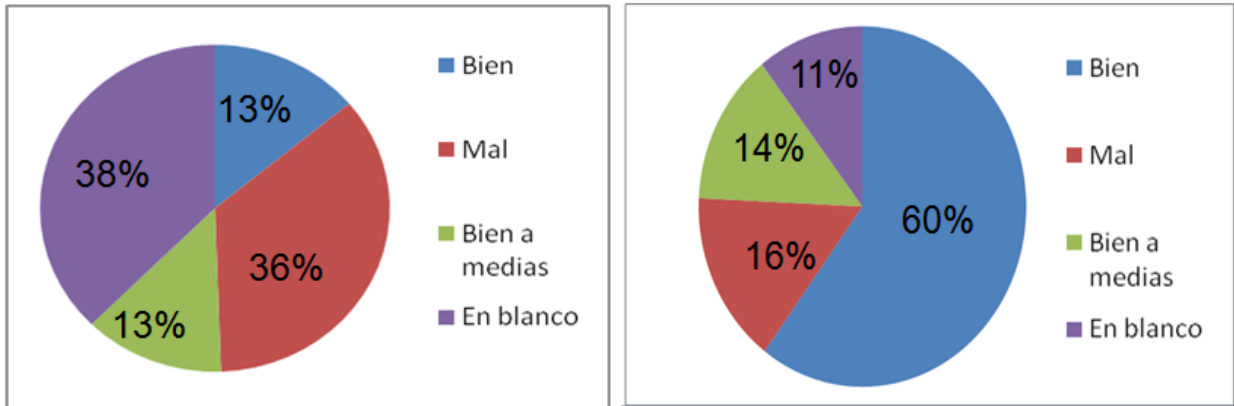

Fig. 2. Resultados de a) Diagnóstico 1 y b) Diagnóstico 2.

\section{Resultados y discusión}

En el análisis de la primera encuesta se puede notar que el tocororo fue identificado en un $100 \%$, a pesar de que en la zona de residencia de los encuestados no se registran avistamientos de esta ave. Esto se explica porque el tocororo es el ave nacional y desde los primeros años de instrucción escolar se enseñan los símbolos patrios.

Las otras aves más identificadas fueron el totí, el tomeguín del pinar y la cartacuba. El primero, por su abundancia en la zona, que es una región arrocera; y el segundo, porque a pesar de su poca existencia en los montes aledaños, es costumbre de los pobladores de los pueblos de campo en Cuba, la captura y cría en jaulas de estas aves, ya sea para escuchar su canto o para destinarlos a peleas, dado que los machos son muy celosos y pelean entre sí por las hembras, principalmente en épocas de cría, aunque las hembras de estas especies también pelean entre sí. En el caso de la cartacuba, además de ser un ave muy llamativa por su colorido y reducido tamaño, últimamente la han 
Juan Carlos Sepúlveda-Peña, Bislandry Vejo-Paula, Carlos A. Villanueva-Hedesa, Juan R. GonzálezGuerra, Alieny González-Alfonso, lanela García-Lau, Gladis Hernández-Horta, Manuel Borrego, Gilberto Socorro, Alberto Amat, Maritza González-Moreno

mostrado en varios programas de la televisión nacional, principalmente en el programa Hábitat. También resalta que en algunos casos la identificación fue parcial, como en el caso del carpintero verde que solo fue identificado como carpintero, y del zunzuncito que lo confundieron con el zunzún común, que no es endémico. En el caso de los gavilanes ocurre algo similar, pues fue señalado como gavilán, pero sin especificar a cuál de los gavilanes se refiere.

En la segunda encuesta se pudo constatar la mejora respecto a los resultados de la primera. En la encuesta inicial solamente el $13.9 \%$ de las respuestas fue completamente acertado, mientras que en la segunda se obtuvo un $60 \%$. Igualmente, el porcentaje de respuestas en blanco disminuyó de un $37.59 \%$ a solo $10.53 \%$. Lo anterior demuestra una gran mejora en el conocimiento de las aves endémicas por parte de los estudiantes participantes.

La primera versión de la aplicación multimedia para Android también fue suministrada a los especialistas de flora y fauna del refugio "Sureste del Inglés". Como parte del proyecto se creó también una página web para la difusión de los resultados en el sitio http://guatini.cubava.cu. Desde dicha página se puede descargar información sobre el proyecto, así como fotografías de aves endémicas tomadas como parte del proyecto.

La primera versión de la aplicación multimedia se limitó a las aves endémicas, dado que solo son 27 especies y uno de los principales problemas con que se enfrentaron los autores del trabajo fue con la recolección de fotos y sonidos de las aves. Existen muchas fotografías de estas aves en internet, pero la mayoría está sujeta a derechos de autor (copyright). Debido a lo anterior, gran parte de las fotografías que conforman la aplicación multimedia fue tomada por los autores del trabajo, y las demás son de autores que previamente han autorizado su uso con fines educativos y conservacionistas sin ánimo de lucro.

En la aplicación multimedia se pueden hacer búsquedas por nombre del ave. Una vez seleccionada el ave, se le muestra al usuario un texto que contiene su nombre común, su nombre científico y una breve descripción de esta, incluyendo aspectos relacionados con la reproducción, nivel de abundancia, 
alimentación y hábitat típicos del ave. También se puede activar el sonido para escuchar el canto de dicha ave (si esta opción está disponible).

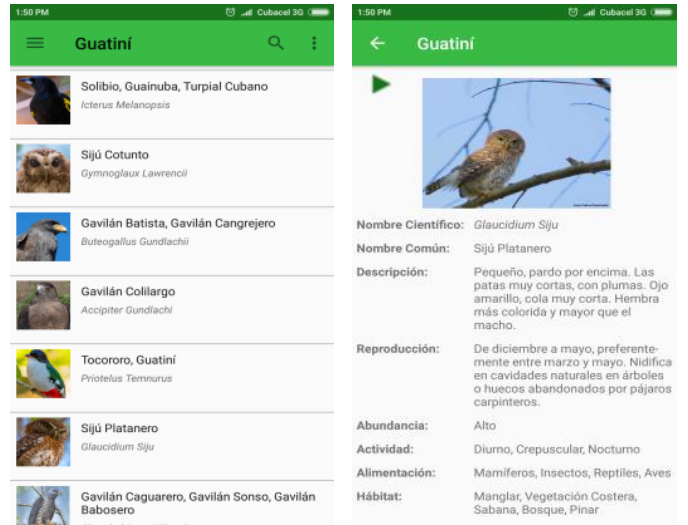

Fig. 3. Imágenes de la aplicación multimedia.

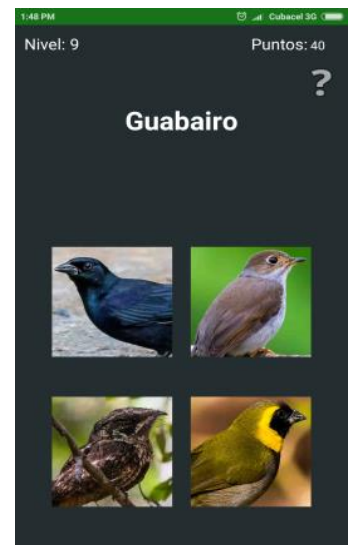

Fig. 4. Imagen del juego.

Los juegos utilizan la misma base de datos que la aplicación multimedia, lo cual posibilita que quien no tenga conocimiento sobre dichas aves, antes de jugar pueda consultar la aplicación. Lo anterior también facilita la personalización del juego a un conjunto de aves en específico, como se mencionó para el caso de la aplicación multimedia.

En general, se pretende que con base en determinada información el usuario sea capaz de identificar al ave. En la Figura 4 se muestra una pantalla del juego donde, a partir del nombre común, el jugador pueda identificar al ave por su imagen. Existen otros niveles del juego en que la identificación debe realizarse desde el nombre científico. Otra variante del juego consiste en mostrar el nombre científico y que el jugador reconozca al ave. Se está trabajando actualmente en una variante en la cual se reconozca al ave por su canto.

\section{Conclusiones}

Al analizar los resultados de las encuestas iniciales y finales realizadas a los estudiantes de sexto y séptimo grado se puede concluir que el desarrollo de aplicaciones informáticas educativas y lúdicas puede servir como una vía alternativa en la educación medioambiental y en el aprendizaje por parte de las nuevas generaciones de las características de la flora y fauna. Bajo el principio 
Juan Carlos Sepúlveda-Peña, Bislandry Vejo-Paula, Carlos A. Villanueva-Hedesa, Juan R. GonzálezGuerra, Alieny González-Alfonso, lanela García-Lau, Gladis Hernández-Horta, Manuel Borrego, Gilberto Socorro, Alberto Amat, Maritza González-Moreno

de que se cuida lo que se ama y solo puede amarse lo que se conoce, incentivar el conocimiento sobre la flora y fauna desde los primeros años de vida es un paso en el cuidado y preservación medioambiental.

También es significativo destacar que los juegos pueden servir no solo para entretener, sino también para educar, y que las TIC pueden ser utilizadas con éxito en el cuidado del medio ambiente, al instruir tanto a las nuevas como a las anteriores generaciones sobre el cuidado de la flora y la fauna.

Los seres humanos somos la especie dominante en este planeta y, por tanto, tenemos la obligación y la responsabilidad de velar por la sobrevivencia del resto de las especies que lo habitan.

\section{Reconocimientos}

Los autores agradecen a las siguientes personas e instituciones, quienes han contribuido con el proyecto "Guatiní": Luis Miguel González, Jesús Sepúlveda, Noel Rodríguez, Rogelio Horta, Emilio Rodríguez, Aslam I. Castellón Maure, Nils Navarro., a la Dirección de Extensión Universitaria de la CUJAE y a los directivos de Refugio de Fauna "Sureste del Inglés".

\section{Referencias}

Aguilar-Mujica, S., Díaz-Fernández, D. F., Clay, R. P., Davidson, I., \& Yépez-Zabala, I. (2009). Cuba. Important Bird Areas America. Quito, Ecuador: BirdLife International.

Birdlife International (2017). Data Zone. Retrieved from: http://www.birdlife.org/datazone/.

Brazuelo, F., \& Cacheiro, M. L. (2015). Estudio de adaptabilidad para dispositivos móviles en plataformas MOOC. RED, Revista de Educación a Distancia, 47(1), 1-13.

Estrada-Betancourt, J. L. (2007). Varias aves cubanas sufren peligro de extinción. Periódico Juventud Rebelde.

Gailey, C. W. (1993). Mediated Messages: Gender, Class, and Cosmos in Home Video Games. The Journal of Popular Culture, 27(1), 81-98. DOI: https://doi.org/10.1111/j.0022-3840.1993.845217931.x.

Lukin, J. G. (2014). Actividades y recursos para la educación ambiental en educación infantil. España: Universidad de La Rioja.

Martínez-Castillo, R. (2010). La importancia de la educación ambiental ante la problemática actual. Revista Electrónica Educare, 14(1), 97-111.

Navarro-Pacheco, N. (2015). Aves endémicas de Cuba. Guía de campo. St. Augustine, FL: Ediciones Nuevos Mundos.

Raffaele, H., Garrido, O. H., Keith, A., \& Raffaele, J. (2003). Birds of the West Indies. New Jersey: Princenton University Press.

Salvat, B. G. (2009). Certezas e interrogantes acerca del uso de los videojuegos para el aprendizaje. Revista Internacional de Comunicación Audiovisual, Publicidad y Literatura, 1(7), 251-264.

Vanderwater, E. A., Wartella, X., Huang, J.H., \& Shim, M. (2007). Digital Childhood: Electronic Media and Technology Use among Infants, Toddlers, and Preschoolers. Pediatrics, 119(5), 1006-1015. DOI: https://doi.org/10.1542/peds.2006-1804. 